\title{
ERROR ESTIMATES FOR THE SEMIDISCRETE FINITE ELEMENT APPROXIMATION OF LINEAR NONLOCAL PARABOLIC EQUATIONS ${ }^{1}$
}

\author{
DENNIS E. JACKSON \\ Florida Institute of Technology \\ Department of Applied Mathematics \\ $150 \mathrm{~W}$. University Blvd. \\ Melbourne, FL 92901 USA
}

\begin{abstract}
Existence and uniqueness are proved for nonlocal (in time) for solutions of linear parabolic partial differential equations. Instead of an initial condition, there is a relation connecting the initial value to values of the solution at other times. $L^{2}$ error estimates are obtained for the semidiscrete approximation of the problem using finite elements in the space variables.
\end{abstract}

Key words: Nonlocal parabolic equations, semidiscrete finite element approximations, error estimates.

AMS (MOS) subject classifications:65M60, 35K20.

\section{INTRODUCTION}

Let $\Omega$ be a bounded open subset of $R^{n}$ with a smooth boundary $\Gamma$. The following nonlocal problem will be considered:

$$
\begin{gathered}
u_{t}+A u=f(x, t) \text { on } \Omega \times(0, T), \\
\left.u\right|_{\Gamma}=0 \\
u(x, 0)+g\left(t_{1}, \ldots, t_{N}, u\right)=\psi(x),
\end{gathered}
$$

where $0<t_{1}<t_{2}<\ldots<t_{N} \leq T, \psi(x) \in L^{2}(\Omega), f(x, t) \in L^{\infty}\left([0, T] ; L^{2}(\Omega)\right)$ and $g\left(t_{1}, \ldots, t_{N}, \cdot\right)$ maps $C^{0}\left([0, T] ; L^{2}(\Omega)\right)$ into $L^{2}(\Omega)$. Also assume $A$ is a strongly elliptic operator defined by

$$
A u=-\sum_{i, j=1}^{n} \frac{\partial}{\partial x_{i}}\left(a_{i j}(x) \frac{\partial u}{\partial x_{j}}\right)+\sum_{i=1}^{n} a_{i}(x) \frac{\partial u}{\partial x_{i}}+a_{0}(x) u
$$

with $a_{i j}(x), a_{i}(x) \in C^{\infty}(\bar{\Omega})$, with

\footnotetext{
${ }^{1}$ Received: February, 1991. Revised: April, 1991.
} 


$$
a(u, u) \geq \sigma\|u\|_{1}^{2}-\lambda_{0}\|u\|^{2}, \quad u \in H_{0}^{1}(\Omega)
$$

where $\sigma>0, \lambda_{0} \in R,\|u\|^{2}=\|u\|_{L^{2}(\Omega)}^{2}=(u, u)$,

$$
a(u, v)=-\sum_{i, j=1}^{n} \int_{\Omega} a_{i j}(x) \frac{\partial u}{\partial x_{i}} \frac{\partial v}{\partial x_{j}} d x+\sum_{i=1}^{n} \int_{\Omega} a_{i}(x) \frac{\partial u}{\partial x_{i}} v d x+\int_{\Omega} a_{0}(x) u v d x
$$

and $H^{s}(\Omega)$ and $H_{0}^{s}(\Omega)$ are the usual Sobolev spaces with norms \|\|$_{s}$. See Adams [1] or Lions [10] for definitions.

Under the above conditions, $A$ with domain $D(A)=H^{2}(\Omega) \cap H_{0}^{1}(\Omega)$ generates an analytic semigroup $S(t)=e^{-A t}$ such that for $a=\sigma-\lambda_{0}$

$$
\|S(t) f\| \leq M e^{-a t}\|f\|
$$

where $M \geq 1$ depends continuously on $\sigma$ and $\lambda_{0}$ in (1.3). See Pazy [5].

The function $u \in C^{0}\left([0, T] ; L^{2}(\Omega)\right)$ is said to be a mild solution of $(1.1)$ if

$$
u(t)=S(t) \psi(x)-S(t) g\left(t_{1}, \quad, t_{N}, u\right)+\int_{0}^{t} S(t-\tau) f(x, \tau) d \tau .
$$

We will assume for $u, v \in C^{0}\left([0, T] ; L^{2}(\Omega)\right)$ of the form $u, v=w$, where

$$
w(t)=S(t) w(0)+\int_{0}^{t} S(t-\tau) f(x, \tau) d \tau
$$

we have the Lipschitz condition

$$
\left\|g\left(t_{1}, \ldots, t_{N}, u\right)-g\left(t_{1}, \ldots, t_{N}, v\right)\right\| \leq \sum_{i=1}^{N} m_{i}\left\|u\left(t_{i}\right)-v\left(t_{i}\right)\right\|
$$

The following are some examples of $g\left(t_{1}, \ldots, t_{N}, u\right):$ If $h_{i}(x) \in C^{\infty}(\bar{\Omega})$, let

$$
g\left(t_{1}, \ldots, t_{N}, u\right)=\sum_{i=1}^{N} h_{i}(x) u\left(t_{i}\right) .
$$

The $m_{i}$ in $(1.8)$ are $m_{i}=\max _{x \in \Omega} x\left|h_{i}(x)\right|$.

Another useful example is

$$
g\left(t_{1}, \ldots, t_{N}, u\right)=\sum_{i=1}^{N} \frac{1}{k_{i}} \int_{t_{i}}^{t_{i}+k_{i}} h_{i}(x, \tau) u(\tau) d \tau
$$

where $k_{i}>0$ and $h_{i}(x, t) \in C^{\infty}(\bar{\Omega} \times[0, T])$. If $u, v$ are as in (1.7) and $t_{i} \leq \tau \leq t_{i}+k_{i}$, then 


$$
\|u(\tau)-v(\tau)\|=\left\|S\left(\tau-t_{i}\right)\left(u\left(t_{i}\right)-v\left(t_{i}\right)\right)\right\| \leq M e^{-a\left(\tau-t_{i}\right)}\left\|u\left(t_{i}\right)-v\left(t_{i}\right)\right\| .
$$

Thus the $m_{i}$ in (1.8) are

$$
m_{i}=\frac{M}{a k_{i}}\left(1-e^{-a k_{i}}\right) \cdot\left(\max _{(x, t) \in \bar{\Omega} \times\left[t_{i}, t_{i}+k_{i}\right]}\left|h_{i}(x, t)\right|\right) .
$$

Nonlocal parabolic problems have been studied by several authors. See Byszewski [25], Chabrowski [6], Hess [7], Kerefov [8], and Vabishchewich [13].

\section{EXISTENCE AND UNIQUENESS FOR NONLOCAL PROBLEMS}

In this section we will prove under the conditions of section $1,(1.6)$ has a unique solution.

Let $W=C^{0}\left([0, T] ; L^{2}(\Omega)\right)$ with norm

$$
\|u\|_{W}=\sup _{0 \leq t \leq T} e^{a t}\|u(t)\|
$$

where $a$ satisfies (1.5). We have the following:

Theorem 2.1: Assume (1.5), (1.8) hold, $\psi(x) \in L^{2}(\Omega)$, and $\sum_{i=1}^{N} m_{i} e^{-a t_{i}}<\frac{1}{M^{2}}$ for $m_{i}$ in (1.8) and $a, M$ in (1.5). Then there is a unique $u$ in $W$ such that $u(t)$ satisfies (1.6).

Proof: Let $\Phi: W \rightarrow W$ be defined by

$$
\begin{gathered}
\Phi v(t)=S(t) \psi(x)-S(t) g\left(t_{1}, \ldots, t_{N}, S(t) v(0)+\int_{0}^{t} S(t-\tau) f(x, \tau) d \tau\right) \\
+\int_{0}^{t} S(t-\tau) f(x, \tau) d \tau
\end{gathered}
$$

for $v \in W$.

We will show $\Phi$ is a contraction mapping on $W$. Let $u, v \in W$. Then

$$
\begin{gathered}
e^{a t}\|\Phi u(t)-\Phi v(t)\| \\
\leq e^{a t} M e^{-a t} \sum_{i=1}^{N} m_{i}\left\|S\left(t_{i}\right)(u(0)-v(0))\right\| \\
\leq M \sum_{i=1}^{N} m_{i} M e^{-a t_{i}}\|u(0)-v(0)\| \\
\leq M^{2}\left(\sum_{i=1}^{N} m_{i} e^{-a t_{i}}\right)\|u-v\|_{W}
\end{gathered}
$$


Thus $\Phi$ is a contraction on $W$, which implies there is a unique $u \in W$ such that $u=\Phi(u)$. Since

and

$$
u(0)=\Phi u(0)=\psi(x)-g\left(t_{1}, \ldots, t_{n}, S(t) u(0)+\int_{0}^{t} S(t-\tau) f(x, \tau) d \tau\right)
$$

it follows that $u(t)$ satisfies (1.6).

$$
u(t)=S(t) u(0)+\int_{0}^{t} S(t-\tau) f(x, \tau) d \tau
$$

Since $S(t)$ has the smoothing property, $S(t) f \in D\left(A^{\frac{\alpha}{2}}\right)$ for $t>0, \alpha \geq 0$ and $f \in L^{2}(\Omega)$, we have the following regularity property:

Corollary 2.2: If the conditions of Theorem 2.1 are satisfied, $\psi(x) \in D\left(A^{\frac{\alpha}{2}}\right), \alpha \geq 0 ;$ $f(x, t) \in L^{\infty}\left([0, T] ; D\left(A^{\mu}\right)\right), \mu=\max \left\{\frac{\alpha}{2}-1+\epsilon, 0\right\}$ for some $\epsilon>0 ;$ and $g\left(t_{1}, \ldots, t_{N}, \cdot\right)$ maps $C^{0}\left((0, T] ; D\left(A^{\frac{\alpha}{2}}\right)\right)$ into $D\left(A^{\frac{\alpha}{2}}\right)$, then the solution $u(t)$ of $(1.6)$ satisfies $u \in C^{0}\left([0, T] ; D\left(A^{\frac{\alpha}{2}}\right)\right)$.

Note: If $\sum_{i=1}^{N} m_{i} e^{-a t}<\frac{1}{M^{2}}$ is not satisfied, there may not be a unique solution. For example, $u_{t}-u_{x x}+\left(a-\pi^{2}\right) u=0$ on $(0,1), u(0, t)=0=u(1, t)$, and $u(x, 0)-e^{-a} u(x, 1)=0$ has solutions $u(x, t)=0$ and $u(x, t)=e^{-a t} \sin \pi x$.

\section{THE SEMIDISCRETE APPROXIMATION}

Let $\left\{V_{h}\right\}$ be a family of finite dimensional subspaces of $H^{1}(\Omega)$ such that for $f \in H^{s}(\Omega), 1 \leq s \leq r$,

$$
\inf _{\chi \in V_{h}}\left\{\|f-\chi\|+h\|f-\chi\|_{1}\right\} \leq c h^{s}\|f\|_{s},
$$

where $c$ is independent of $h$. $u=e^{\lambda_{0} t} W$.

In this section we will assume (1.3) is satisfied with $\lambda_{0}=0$. If this is not the case, let

For fixed $\epsilon>0$, assume $A_{h}: V_{h} \rightarrow V_{h}$ satisfies

$$
\left(A_{h} f_{h}, f_{h}\right) \geq \sigma^{\prime}\left\|f_{h}\right\|^{2} \text { if } f_{h} \in V_{h},
$$

where $0<\sigma-\epsilon<\sigma^{\prime} \leq \sigma$,

$$
\left(A_{h} f_{h}, g_{h}\right) \leq c\left\|f_{h}\right\|_{1}\left\|g_{h}\right\|_{1} \text { for all } f_{h}, g_{h} \in V_{h}
$$

and 


$$
\left\|\left(P_{h} A^{-1}-A_{h}^{-1} P_{h}\right) f\right\| \leq c h^{\alpha+2}\left\|A^{\frac{\alpha}{2}} f\right\|, \quad 0 \leq \alpha \leq r-2
$$

where $P_{h}$ is the $L^{2}$ projection of $L^{2}(\Omega)$ onto $V_{h}$.

Conditions (3.2),(3.3) and (3.4) are satisfied with $\sigma^{\prime}=\sigma$ if the standard Galerkin method is used with $V_{h} \in H_{0}^{1}(\Omega)$ and $A_{h}$ is defined by

$$
\left(A_{h} f_{h}, g_{h}\right)=\left(A f_{h}, g_{h}\right), \quad f_{n}, v_{n} \in V_{n}
$$

The conditions are also satisfied if Nitsche's method is used, where $V_{h} \subseteq H^{1}(\Omega),\left.V_{h}\right|_{\Gamma} \subseteq H^{1}(\Gamma)$, for $2 \leq s \leq r$,

$$
\inf _{\chi \in V_{h}}\left\{\|f-\chi\|+h\|f-\chi\|_{1}+h^{\frac{1}{2}}\|f-\chi\|_{L^{2}(\Gamma)}+h^{\frac{3}{2}}\|f-\chi\|_{H^{1}(\Gamma)}\right\} \leq c h^{s}\|f\|_{s}
$$

and $A_{h}: v_{h} \rightarrow v_{n}$ is defined by

$$
\left(A_{h} f_{h}, g_{h}\right)=a\left(f_{h}, g_{h}\right)-\left(\frac{\partial f_{h}}{\partial n}, g_{h}\right)_{L^{2}(\Gamma)}-\left(f_{h}, \frac{\partial g_{h}}{\partial n}\right)_{L^{2}(\Gamma)}+\beta h^{-1}\left(f_{h}, g_{h}\right)_{L^{2}(\Gamma)}
$$

for $\beta$ large enough such that (3.2) holds. See Lasiecka [9].

We will first show the following nonlocal system on $V_{h}$ has a unique solution for $0 \leq t \leq T$ :

$$
\begin{gathered}
u_{h}^{\prime}(t)+A_{h} u_{h}=P_{h} f(x, t), \\
u_{h}(0)+P_{h} g\left(t_{1}, \ldots, t_{N}, u_{h}\right)=P_{h} \psi .
\end{gathered}
$$

Let $S_{h}(t)=e^{-A_{h} t}$, then (3.5) is equivalent to

$$
u_{h}(t)=S_{h}(t) P_{h} \psi-S_{h}(t) P_{n} g\left(t_{1}, \ldots, t_{N}, u_{h}\right)+\int_{0}^{t} S_{h}(t-\tau) P_{h} f(x, \tau) d \tau .
$$

Since $\left\|e^{-A_{h} t} f_{h}\right\| \leq M \sigma^{\prime} e^{-\sigma^{\prime} t}<\frac{1}{M_{\sigma^{\prime}}^{2}}$, where $\underset{\sigma^{\prime} \rightarrow \sigma^{\prime}}{\lim _{\sigma^{\prime}}}=M$, we can find $\epsilon>0$ for

(3.2) and $\delta>0$ such that if $m_{i}^{\prime}=m_{i}+\delta$ and $\sum_{i=1}^{N} m_{i} e^{-\sigma^{\prime} t_{i}}<\frac{1}{M^{2}}$, then

$$
\sum_{i=1}^{N} m_{i}^{\prime} e^{-\sigma^{\prime} t_{i}}<\frac{1}{M_{\sigma^{\prime}}^{2}}
$$

Thus by a similar proof to that of Theorem 1.1, we can prove the following: 
Theorem 3.1: Assume the conditions in Theorem 1.1 are satisfied and $V_{h}$ and $A_{h}$ satisfy (3.1)-(3.4), where $\sigma^{\prime}$ from (3.2) is such that (3.7) holds and

$$
\left\|P_{h}\left(g\left(t_{1}, \ldots, t_{N}, u_{h}\right)-g\left(t_{1}, \ldots, t_{N}, v_{h}\right)\right)\right\| \leq \sum_{i=1}^{N} m_{i}^{\prime}\left\|u_{h}\left(t_{i}\right)-v_{h}\left(t_{i}\right)\right\|
$$

for $u_{h}, v_{h}=w_{n}$ of the form $w_{h}(t)=S_{h}(t) w_{h}(0)+\int_{0}^{t} S_{h}(t-\tau) P_{h} f(x, \tau) d \tau$. Then there is a unique solution $u_{h}(t)$ of (3.6) such that $u_{h} \in C^{0}\left([0, T] ; V_{h}\right)$.

Since $\left\|P_{h}\left(h(x) f_{h}\right)\right\| \leq\left(\sup _{x \in \Omega}|h(x)|\right)\left\|f_{h}\right\|$ for $f_{h} \in V_{h}$, if $\sigma^{\prime}$ is close enough to $\sigma$, then $g$ defined in (1.9) and (1.10) satisfy (3.8).

Under the assumptions (3.1)-(3.4), we have for $a \leq s \leq r$ and $f \in D\left(A^{\frac{\alpha}{2}}\right), 0 \leq \alpha \leq s$ the condition

$$
\left\|\left(S(t)-S_{h}(t) P_{h}\right) f\right\| \leq \frac{C h^{s}}{\frac{s-\alpha}{2}}\left\|A^{\frac{\alpha}{2}} f\right\|
$$

and for $f(x, t) \in L^{\infty}\left(0, T ; D\left(A^{\frac{\alpha^{\prime}}{2}}\right)\right), \quad 0 \leq \alpha^{\prime} \leq r-2$

$$
\left\|\int_{0}^{t}\left(S(t-\tau)-S_{h}(t-\tau) P_{h}\right) f(x, \tau) d \tau\right\| \leq C h^{\alpha^{\prime}+2} \ln \left(\frac{1}{h}\right)\|f\|_{L^{\infty}\left(0, T ; D\left(A^{\frac{\alpha^{\prime}}{2}}\right)\right)} .
$$

See for example Lasiecka [9] or Thomée [12].

We can now prove similar error estimates for the semidiscrete approximation to the nonlocal problems.

Theorem 3.2: Let the assumptions of Theorems 1.1 and 3.1 be satisfied, and let the hypotheses of Corollary 2.2 be satisfied for $\alpha \leq r, f(x, t) \in L^{\infty}\left(0, T ; D\left(A^{\frac{\theta}{2}}\right)\right), \theta=\max \left\{\mu, \alpha^{\prime}\right\}$, $0 \leq \alpha^{\prime} \leq r-2$, and for $u, v \in C^{0}\left(\left[t_{1}, T\right], L^{2}(\Omega)\right)$,

$$
\left\|g\left(t_{1}, \ldots, t_{N}, u\right)-g\left(t_{1}, \ldots, t_{N}, v\right)\right\| \leq k\|u-v\|_{L^{\infty}\left(t_{1}, T ; L^{2}(\Omega)\right)}
$$

Also assume that $u(t)$ is the solution of (1.6) and $u_{h}(t)$ is the solution to (3.6) for $\alpha \leq s \leq r$. Then

$$
\left\|u(t)-u_{h}(t)\right\| \leq C h^{s}\left(\frac{1}{t^{\frac{s-\alpha}{2}}}+1\right)+C h^{\alpha^{\prime}+2} \ln \left(\frac{1}{h}\right)\|f\|_{\left.\left.L_{\left(0, T ; D\left(A^{\infty}\right.\right.}^{\frac{\alpha^{\prime}}{2}}\right)\right)}
$$


Proof: We have

$$
\begin{aligned}
& \left\|u(t)-u_{h}(t)\right\| \leq\left\|\left(S(t)-S_{h}(t) P_{h}\right) \psi\right\|+\left\|\left(S(t)-S_{h}(t) P_{h}\right) g\left(t_{1}, \ldots t_{N}, u\right)\right\| \\
& +\left\|S_{h}(t) P_{h}\left(g\left(t_{1}, \ldots, t_{N}, u\right)-g\left(t_{1}, \ldots, t_{N}, u_{h}\right)\right)\right\| \\
& +\left\|\int_{0}^{t}\left(S(t-\tau)-S_{h}(t-\tau) P_{h}\right) f(x, \tau) d \tau\right\| \\
& \leq \frac{C h^{s}}{t^{\frac{s-\alpha}{2}}}\left(\left\|A^{\frac{\alpha}{2}} \psi\right\|+\left\|A^{\frac{\alpha}{2}} g\left(t_{1}, \ldots, t_{N}, u\right)\right\|\right)+C h^{\alpha^{\prime}+2} \ln \frac{1}{h}\|f\|_{L^{\infty}\left(0, T ; D\left(A^{\frac{\alpha^{\prime}}{2}}\right)\right)} \\
& +M_{\sigma^{\prime}} e^{-\sigma^{\prime} t}\left\|g\left(t_{1}, \ldots, t_{N}, u\right)-g\left(t_{1}, \ldots, t_{N}, u_{h}\right)\right\| \text {. }
\end{aligned}
$$

Since $A_{h}$ is bounded, $S_{h}(-t)=e^{A_{h} t}$ exists. Let $t \geq t_{1}$, then

$$
\begin{aligned}
& \left\|g\left(t_{1}, \ldots, t_{N}, u\right)-g\left(t_{1}, \ldots, t_{N}, u_{h}\right)\right\| \\
& \leq\left\|g\left(t_{1}, \ldots, t_{N}, u\right)-g\left(t_{1}, \ldots, t_{N}, S_{h}\left(t-t_{1}\right) P_{h} S\left(t_{1}\right) u(0)+\int_{0}^{t} S_{h}(t-\tau) P_{h} f(x, \tau) d \tau\right)\right\| \\
& +\| g\left(t_{1}, \ldots, t_{N}, S_{h}(t)\left(S_{h}\left(-t_{1}\right) P_{h} S\left(t_{1}\right) u(0)\right)+\int_{0}^{t} S_{h}(t-\tau) P_{h} f(x, \tau) d \tau\right) \\
& -g\left(t_{1}, \ldots, t_{N}, u_{h}\right) \| \\
& \leq k \underset{t_{1} \leq t \leq T}{\sup }\left(\left\|\left(S\left(t-t_{1}\right)-S_{h}\left(t-t_{1}\right) P_{h}\right) S\left(t_{1}\right) u(0)\right\|+\left\|\int_{0}^{t}\left(S(t-\tau)-S_{h}(t-\tau) P_{h}\right) f(x, \tau) d \tau\right\|\right. \\
& +\sum_{i=1}^{N} m_{i}^{\prime}\left\|S_{h}\left(t_{i}\right)\left(S_{h}\left(-t_{1}\right) P_{h} S\left(t_{1}\right) u(0)\right)-S_{h}\left(t_{i}-t_{1}\right) S_{h}\left(t_{1}\right) u_{h}\right\| \\
& \leq C h^{s}\left\|A^{\frac{s}{2}} S\left(t_{1}\right) u(0)\right\|+C h^{\alpha^{\prime}+2} \ln \frac{1}{h}\|f\| \underset{L^{\infty}\left(0, T ; D\left(A^{\frac{\alpha^{\prime}}{2}}\right)\right)}{ } \\
& +\sum_{i=1}^{N} m_{i}^{\prime} M_{\sigma^{\prime}} e^{-\sigma^{\prime}\left(t_{i}-t_{1}\right)}\left\|S\left(t_{1}\right) u(0)-S_{h}\left(t_{1}\right) u_{h}(0)\right\|
\end{aligned}
$$

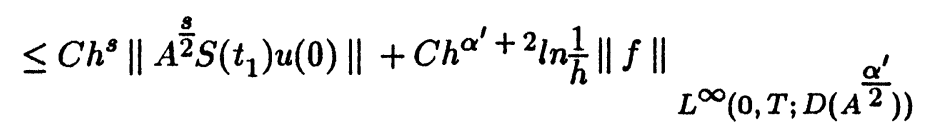

$$
\begin{aligned}
& +\sum_{i=1}^{N} m_{i} M_{\sigma^{\prime}} e^{-\sigma^{\prime}\left(t_{i}-t_{1}\right)}\left(\left\|u\left(t_{1}\right)-u_{h}\left(t_{1}\right)\right\|+\left\|\int_{0}^{t_{1}}\left(S(t-\tau)-S_{h}(t-\tau) P_{h}\right) f(x, \tau) d \tau\right\|\right)
\end{aligned}
$$




$$
\begin{gathered}
\leq C h^{s}\left\|A^{\frac{s}{2}} S\left(t_{1}\right) u(0)\right\|+C h^{\alpha^{\prime}+2} \ln \frac{1}{h}\|f\|{ }_{L^{\infty}\left(0, T ; D\left(A^{\frac{\alpha^{\prime}}{2}}\right)\right)} \\
+\sum_{i=1}^{N} m_{i} M_{\sigma^{\prime}} e^{-\sigma^{\prime}\left(t_{i}-t_{1}\right)}\left\|u\left(t_{1}\right)-u_{h}\left(t_{1}\right)\right\|
\end{gathered}
$$

Let $t=t_{1}$ in (3.13), then

$$
\begin{aligned}
\left\|u\left(t_{1}\right)-u_{h}\left(t_{1}\right)\right\| \leq & C\left(\frac{h^{s}}{t_{1}^{\frac{s-\alpha}{2}}}+1\right)+C h^{\alpha^{\prime}+2} \ln \left(\frac{1}{h}\right)\|f\|{ }_{L}^{\infty}\left(0, T ; D\left(A^{\frac{\alpha^{\prime}}{2}}\right)\right) \\
& +M_{\sigma^{\prime}}^{2} \sum_{i=1}^{N} m_{i}^{\prime} e^{-\sigma^{\prime} t_{i}}\left\|u\left(t_{1}\right)-u_{h}\left(t_{1}\right)\right\| .
\end{aligned}
$$

Since $M_{\sigma^{\prime}}^{2} \sum_{i=1}^{N} m_{i}^{\prime} e^{-\sigma^{\prime} t_{i}}<1,(3.12)$ holds for $t=t_{1}$. Therefore the theorem follows from (3.13) and (3.15).

\section{REFERENCES}

[1] R.A. Adams, "Sobolev Space", Academic Press, New York, (1975).

[2] L. Byszewski, "Strong maximum and minimum principles for parabolic problems with nonlocal inequalities", Zeitschrift für Angewandte Mathematik und Mechanik 70.3, pp. 202206, (1990).

[3] L. Byszewski, "Strong maximum principles for parabolic nonlinear problems with nonlocal inequalities together with integrals", Journal of Applied Mathematics and Stochastic Analysis 3.5, pp. 65-79, (1990).

[4] L. Byszewski, "Strong maximum principles for parabolic nonlinear problems with nonlocal inequalities together with arbitrary functionals", Journal of Mathematical Analysis and Applications 1.56, (1991), (to appear).

[5] L. Byszewski, "Theorems about the existence and uniqueness of a semilinear evolution nonlocal Cauchy problem", Journal of Mathematical Analysis and Applications, (1991), (to appear).

[6] J. Chabrowki, "On nonlocal problems for parabolic equations", Nagoya Math. J. 93, pp. 109-131, (1984).

[7] P. Hess, "Spatial homogeneity of stable solutions of some periodic-parabolic problems with Neumann boundary conditions", J. of Differential and Integral Equations 68, pp. 320-331, (1987).

[8] A.A. Kerefov, "Nonlocal boundary value problems for parabolic equations", (in Russian), Differentsial'nye Uravnenija 15, pp. 74-78, (1979).

[9] I. Lasiecka, "Convergence estimates for semidiscrete approximations of nonself adjoint parabolic equations", SIAM J. Numer. Anal. 21, No. 5, pp. 894-909, (1984). 
[10] J.L. Lions, “Non-Homogeneous Boundary Value Problems and Applications", Vol. 1, Springer-Verlag, New York, (1972).

[11] A. Pazy, "Semigroups of Linear Operators and Applications to Partial Differential Equations", Springer-Verlag, New York, (1983).

[12] V. Thomée, "Galerkin Finite Element Methods for Parabolic Problems", Lecture Notes in Mathematics, Vol. 1054, Springer-Verlag, New York, (1984).

[13] P.N. Vabishchevich, "Nonlocal parabolic problems and the inverse heat-conduction problem", (in Russian), Differentsial'nye Uravnenija 17, pp. 1193-1199, (1981). 


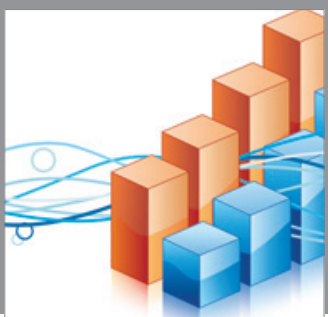

Advances in

Operations Research

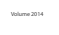

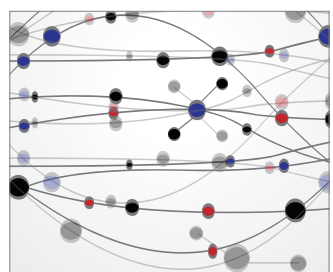

\section{The Scientific} World Journal
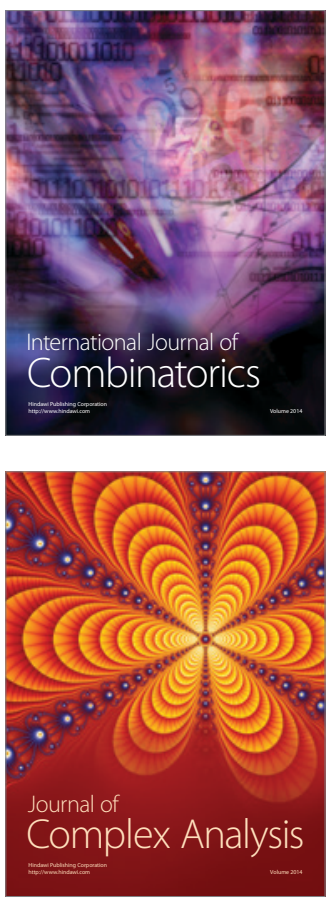

International Journal of

Mathematics and

Mathematical

Sciences
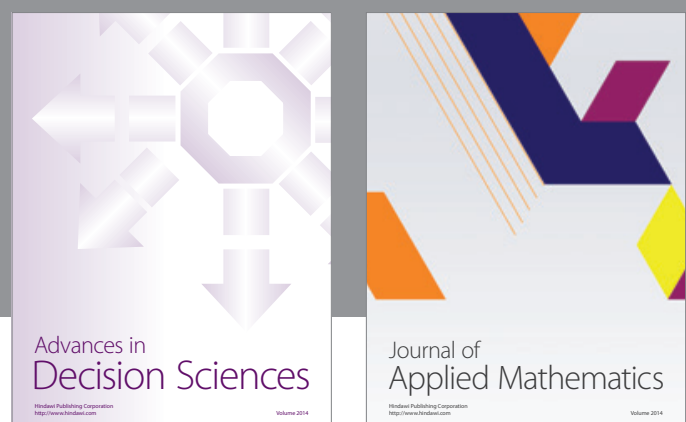

Journal of

Applied Mathematics
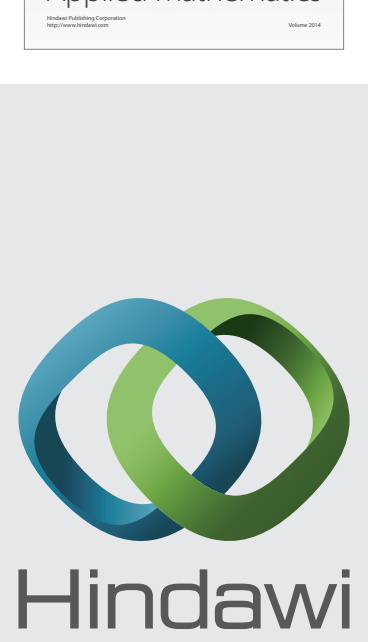

Submit your manuscripts at http://www.hindawi.com
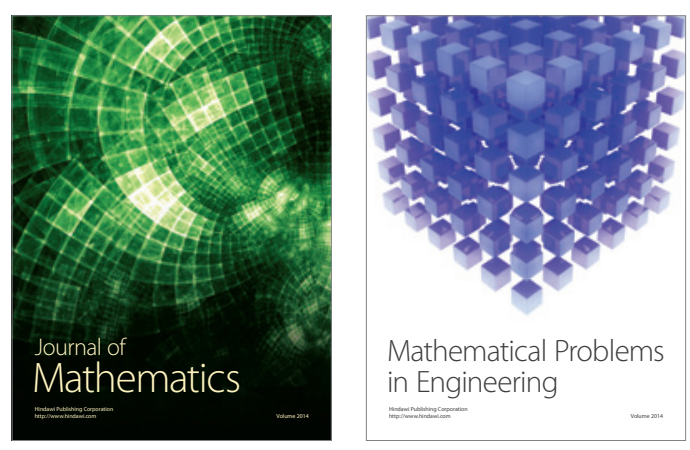

Mathematical Problems in Engineering
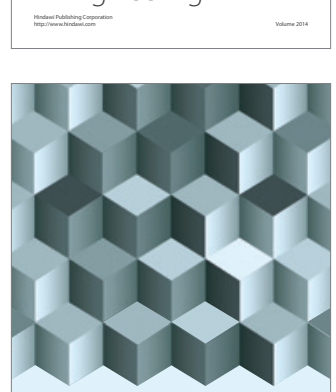

Journal of

Function Spaces
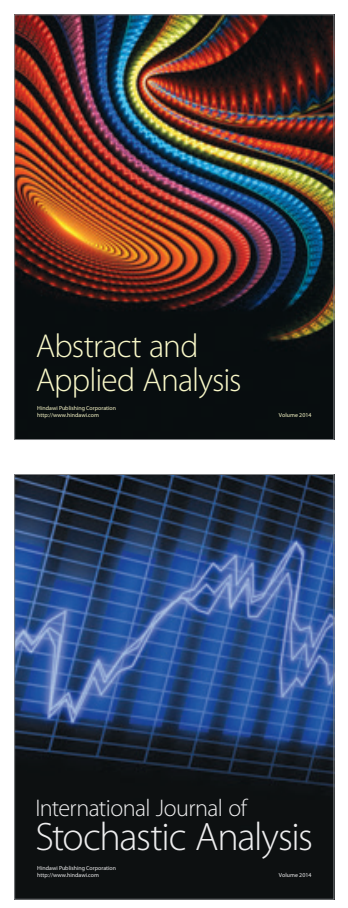

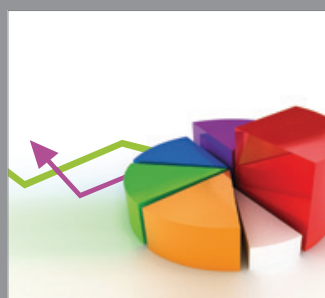

ournal of

Probability and Statistics

Promensencen
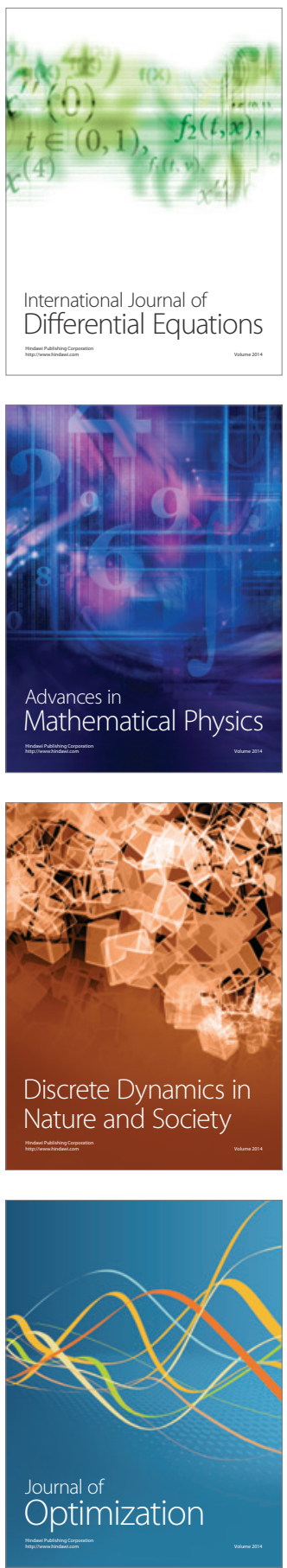